\title{
Procesos gravitacionales en zonas de ladera, aplicándolos a modelos de predicción real con archivos raster y SIG, Alcaldía Álvaro Obregón, Ciudad de México
}

\author{
Oscar Daniel Rivera González¹
}

Recibido el 30 de marzo de 2019; aceptado el 25 de abril de 2019

\section{Resumen}

El motivo para realizar el siguiente trabajo de investigación, es la generación de cartografía con un nivel de detalle suficiente para generar algún tipo de predicción, aproximadamente de tres a cuatro metros de tolerancia de error, sustentado con base en puntos de control tomados con Sistema de Posicionamiento Global (GPS), Ilevándolo a la realidad para poder generar prevención, atención, gestión o coordinación, por parte de las autoridades de las alcaldías, estatales y federales, sobre la presencia de asentamientos humanos permitidos y no permitidos, en zonas urbanas al norte de la Alcaldía Álvaro Obregón, en la cual, se observa algún grado de vulnerabilidad geofísica, geomorfológica, geológica o topográfica. Por otra parte, el alto nivel de marginación por parte de las familias, que habitan dichas zonas, situadas en zonas de montaña como: laderas, cauces de ríos o barrancas, por lo que son un peligro constante por su pronunciado grado de inclinación y al ocurrir algún proceso gravitacional, arrastra o acarrea la cimentación de las construcciones existentes y lo que tenga a su paso, más aún en temporada de lluvias, movimientos sísmicos, colapsos de techos de minas, derrumbes, lo cual ocasiona pérdida de vidas humanas.

Por otra parte, esto da como resultado un deterioro medioambiental en torno al ecosistema original y que arbitrariamente se modificó con el paso de los años, con esta desarticulada urbanización reglamentada y no reglamentada por la Alcaldía Álvaro Obregón, ya que al realizarse este tipo de edificaciones en zonas no aptas para su habitabilidad, inclusive en áreas naturales protegidas ahora urbanizadas. Por las problemáticas antes mencionadas, se elimina o reemplaza la cobertura vegetal, observándose la nula filtración del agua pluvial por la compactación del suelo en cuanto a la utilización de materiales para construcción y recientemente se encontró en trabajo de campo, piedra pómez, la cual es muy endeble cuando se encuentra hidratada.

Geógrafo, Especialista en Economía Ambiental y Ecológica, Maestría en Urbanismo, Estudiante de Doctorado en Urbanismo, Universidad Nacional Autónoma de México (unam). Correo electrónico: oscardanieldanyboy@hotmail.com 
Algo de suma importancia, es que las personas que habitan estas zonas con algún grado de vulnerabilidad, rellenan o rellenaron de alguna forma pequeñas cavidades o minas con material de cascajo, residuos orgánicos, residuos sólidos o basura para la nivelación del suelo, lo cual solo incrementa las graves consecuencias, haciéndolo aún más inestable.

Se tratará de dar solución a lo antes mencionado, con la utilización de archivos tipo Raster extensión TIFF, GEOTIFF y BIL, obtenidos de la página electrónica de la National Aeronautics and Space Administration (NASA): https://vertex.daac.asf. alaska.edu/, y del Instituto Nacional de Estadística y Geografía (INEGI): http://www. beta.inegi.org.mx/temas/mapas/relieve/continental/, con base en estos archivos, se generaron modelos de predicción $3 \mathrm{~d}$ con ayuda de Sistemas de Información Geográfica (SIG) y visitas a campo.

Palabras clave: Procesos gravitacionales, zonas de ladera, modelos de predicción, vulnerabilidad y Sistemas de Información Geográfica (SIG).

\section{Abstract}

The reason for doing the following research work is the generation of cartography and sufficient level of detail to generate some kind of prediction, approximately three to four meters error tolerance, based on control points taken with Positioning System Global (GPS), all this taking it to reality to be able to generate, prevention, attention, management or coordination, on the part of the authorities of the acaldías, state and federal, on the presence of human settlements allowed and not allowed, in urban areas north of the Alcaldía Álvaro Obregón, in which, some degree of geophysical, geomorphological, geological or topographical vulnerability is observed, on the other hand the high level of marginalization on the part of the families, which inhabit these zones, located in mountain areas as they are; slopes, riverbeds or ravines, for what they are, a constant danger due to its pronounced degree of inclination and when some gravitational process occurs, it drags or carries the foundations of existing constructions and what is in its way, even more so in season of rains, seismic movements, collapses of mine roofs, landslides, which causes loss of human life.

On the other hand, this results in an environmental deterioration around the original ecosystem and that was arbitrarily modified over the years, with this disarticulated urbanization regulated and not regulated by the Alvaro Obregon City Hall, since when this type was carried out of buildings in areas not suitable for habitability, including natural protected areas now urbanized, for the problems mentioned above, the vegetation cover is eliminated or replaced, observing the non-filtration of rainwater by the compaction of the soil in terms of the use of materials for construction and recently found in field work, pumice stone, which is very weak when it is hydrated.

Something of utmost importance, is that the people who live in these areas with some degree of vulnerability, fill or fill in some way, small cavities or mines 
with gravel material, organic waste, solid waste or trash for leveling the soil, which it only increases and brings serious consequences, making it even more unstable.

It will try to solve the aforementioned, with the use of files type Raster extension TIFF, GEOTIFF and BIL, obtained from the website of the National Aeronautics and Space Administration (NASA): https: //vertex.daac.asf. alaska.edu/, and the National Institute of Statistics and Geography (INEGI): http://www.beta. inegi.org.mx/temas/mapas/relieve/continental/, based on these files, models of $3 d$ prediction with the help of Geographic Information Systems (GIS) and field visits.

Key words: Gravitational processes, slope zones, prediction models, vulnerability and Geographic Information Systems (GIS).

\section{Resumo}

A razão para o seguinte trabalho de pesquisa é a geração de mapeamento e nível suficiente para gerar algum tipo de previsão cerca de três a quatro metros de detalhe tolerância de erro, apoiada com base em pontos de controlo tomadas com Sistema de Posicionamento global (GPS), tudo levando-o a realidade a fim de gerar, prevenção, assistência, gestão ou coordenação por parte das autoridades do acaldías, estadual e federal, com a presença de assentamentos humanos permitidos e não permitidos em áreas urbanas ao norte do prefeito Alvaro Obregon, nos quais algum grau de geofísica, geomorfologia, a vulnerabilidade geológica ou topográfica se observa, por outro lado, o alto nível de marginalização pelas famílias que habitam essas áreas, localizado em áreas montanhosas como elas são; taludes, leitos de rios ou valas, por isso são um perigo constante para a sua inclinação pronunciada e um processo gravitacional ocorrer, arrasta ou transporta as fundações de edifícios existentes e que tem o seu caminho, especialmente na época chuva, terremotos, o meu telhado desmorona, deslizamentos de terra, causando perda de vida.

Por outro lado, isso resulta em uma deterioração ambiental em torno do ecossistema original e que foi arbitrariamente modificado ao longo dos anos, com essa urbanização desarticulada regulamentada e não regulada pela Prefeitura de Álvaro Obregón, desde quando esse tipo era realizado. de edificações em áreas não habitáveis, incluindo áreas naturais protegidas hoje urbanizadas, para os problemas citados acima, a cobertura vegetal é eliminada ou substituída, observando a não filtração da água da chuva pela compactação do solo em termos do uso de materiais para construção e recentemente encontrados em trabalhos de campo, pedra-pomes, que é muito fraca quando é hidratada.

Algo da maior importância, é que as pessoas que vivem nestas áreas com algum grau de vulnerabilidade, preenchem ou preenchem de alguma forma, pequenas cavidades ou minas com material de cascalho, resíduos orgânicos, resíduos sólidos ou lixo para nivelamento do solo, que só aumenta e traz graves consequências, tornando ainda mais instável. 
Ele tentará resolver o problema acima mencionado, com o uso de arquivos do tipo Raster extension TIFF, GEOTIFF e BIL, obtidos no site da NASA: https://vertex. daac.asf. alaska.edu/, e do Instituto Nacional de Estatística e Geografia (INEGI): http://www.beta.inegi.org.mx/temas/mapas/relieve/continental/, com base nesses arquivos, modelos de Previsão 3D com a ajuda de Sistemas de Informações Geográficas (GIS) e visitas de campo.

Palavras-chave: Processos gravitacionais, zonas de declividade, modelos de previsão, vulnerabilidade e Sistemas de Informação Geográfica (SIG).

\section{Introducción}

La Alcaldía Álvaro Obregón se ubica al poniente de la Ciudad de México, en las coordenadas: $19^{\circ} 14^{\prime} ; 19^{\circ} 25^{\prime} \mathrm{N}$ y $99^{\circ} 10^{\prime} ; 99^{\circ} 20^{\prime} \mathrm{W}$, su extensión territorial es de 96.17 km² $^{2}$, situada sobre las laderas del volcán San Miguel perteneciente a la Sierra de las Cruces. La geología de la alcaldía está constituida por grandes derrames de lavas fuertemente accidentados y fracturados, combinado con las pendientes, dándose a lo largo de la alcaldía, zonas susceptibles a presentar procesos de ladera (Figuras 1 y 2 ) y un material recientemente encontrado en trabajo de campo: la piedra pómez, mismo que se convierte en un tipo de masa con la precipitación, la cual hace más vulnerables sitios dentro de la zona de estudio, así como a las edificaciones existentes.

Por lo que este análisis se enfoca en la problemática de procesos gravitacionales, también conocidos como procesos de remoción en masa, en zonas marginadas dentro de la zona Norte de la Alcaldía Álvaro Obregón, específicamente en las colonias: Desarrollo Urbano, Desarrollo Urbano el Pirú, Presidentes, Tejocote y Lomas de Capúla (Figura 2) dentro las cuales converge la zona de estudio de este artículo de investigación.

Las amenazas, junto con las condiciones imperantes de exposición, vulnerabilidad y resiliencia, causan riesgo. Las amenazas, cuando se materializaron como un evento físico concreto, pueden desencadenar una escalada de condiciones ya de por si inseguras, en un estado de crisis o emergencia (Alcántara, 2016).

La urbanización en zonas no aptas para la construcción, se debe en mayor medida al indebido crecimiento poblacional que se da en la Ciudad de México específicamente en la Alcaldía Álvaro Obregón, las autoridades ya sean locales, estatales o federales, debieron ubicar zonas con potencial de urbanizacion y en caso de no ser así, tratar de aminorar dicha problemática con algún tipo de mapeo, basado en modelos de predicción, seguido de una política pública.

Las cinco colonias mencionadas anteriormente no son de interés para el Gobierno mexicano, hasta cierto punto son zonas olvidadas por su grado de marginación económica y social, enfocándose solamente en la zona de Santa Fe 


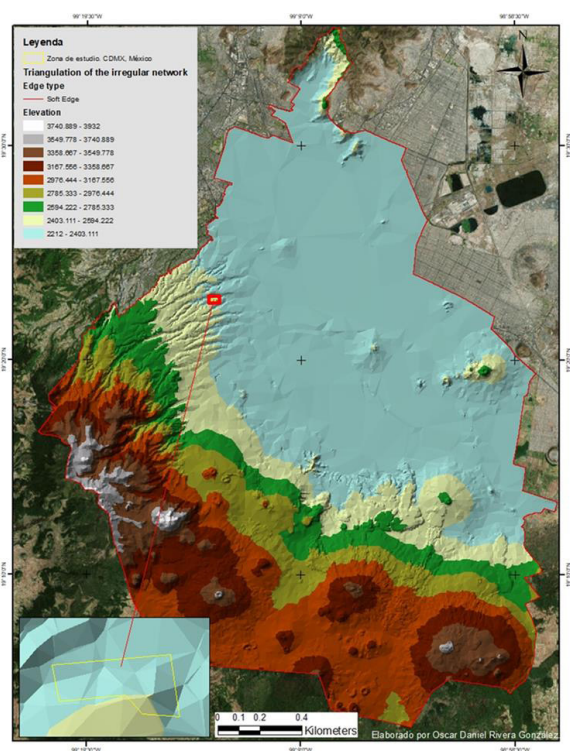

Figura 1. Elaborada con base en datos Raster del Instituto Nacional de Estadística y Geografía (INEGI). Elaborado con Software ArcGis.

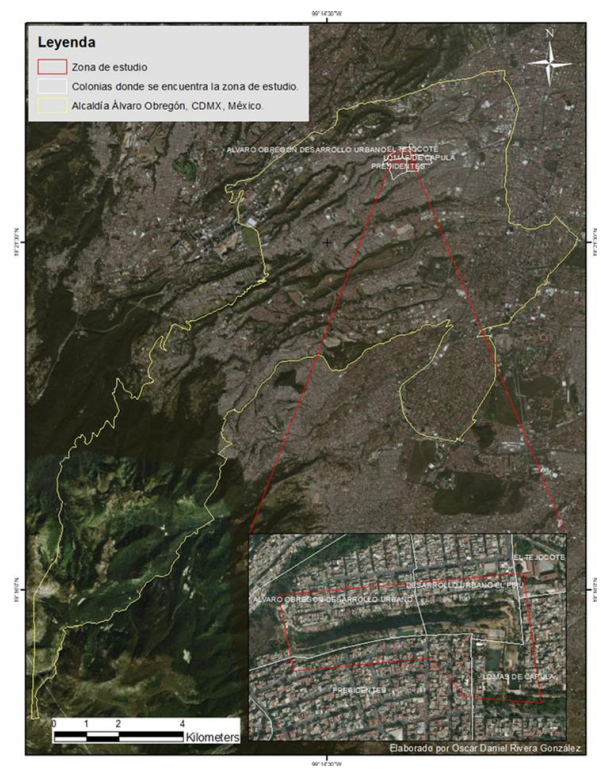

Figura 2. Elaborada con base en datos Raster del Instituto Nacional de Estadística y Geografía (INEGI). Elaborado con Software ArcGis. 
y alrededores, las cuales dejan derramas económicas altas, ya que se encuentran situados allí varios corporativos y empresas particulares.

Existen zonas poco atendidas dentro de la Alcaldía Álvaro Obregón, las cuales en temporada de lluvias, de mayo a noviembre se ven afectadas, ya que se activan de nuevo los ríos desecados, generando diversas problemáticas, como procesos de remoción en masa, reblandecimiento de tierra e inundaciones, todo esto con base a entrevistas con los habitantes de las cinco colonias mencionadas.

Este artículo investigó la falta de una adecuada planeación para la gestión del riesgo, con la finalidad de prevenirlo y atenderlo eficientemente, asimismo se generó como ejemplo, un modelo de predicción basado en Sistemas de Información Geográfica (SIG) y modelos matemáticos simples para poder ser utilizados en un futuro por parte de la acaldía, lo cual permitirá proteger a los habitantes de estas regiones tan marginadas y olvidadas, así como de la infraestructura urbana de los mismos; cabe mencionar que se permitió una indebida construcción, por parte de las inmobiliarias, como de autoconstrucción por parte de la población, en zonas susceptibles y vulnerables a procesos de remoción en masa, algunas dadas por la inundabilidad, geomorfología (Figuras 3-5), topografía, reblandecimiento de tierra y movimientos telúricos, siendo estas zonas no propicias para la construcción y cobrando por lo tanto vidas humanas.

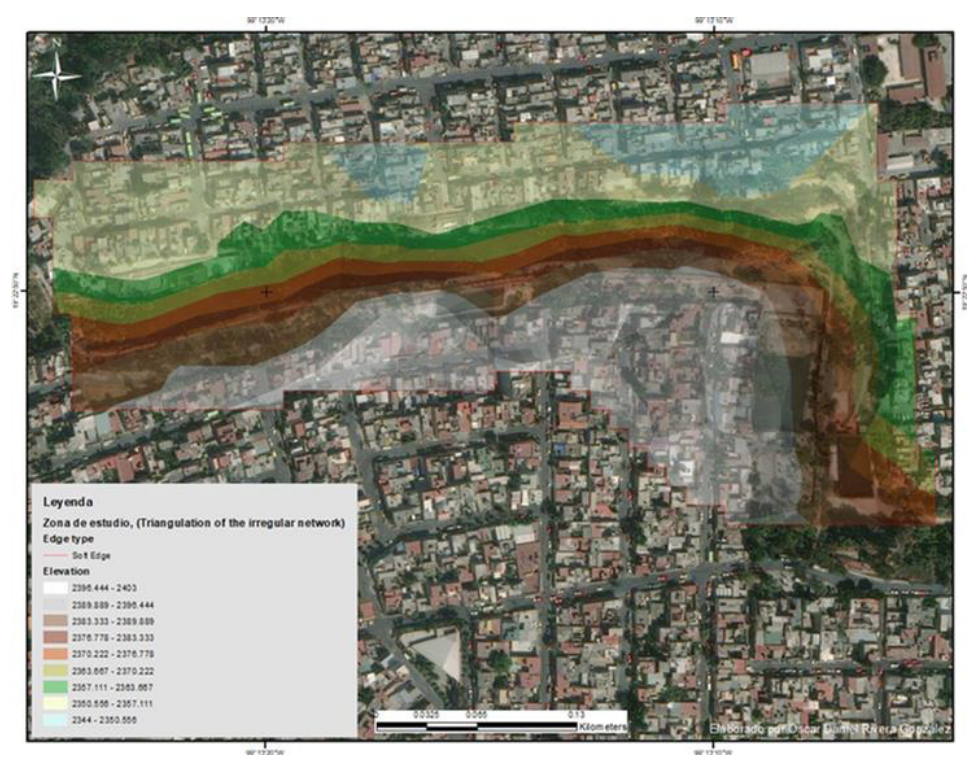

Figura 3. Elaborada con base en datos Raster de la National Aeronautics and Space Administration (NASA). Elaborado con Software ArcGis. 


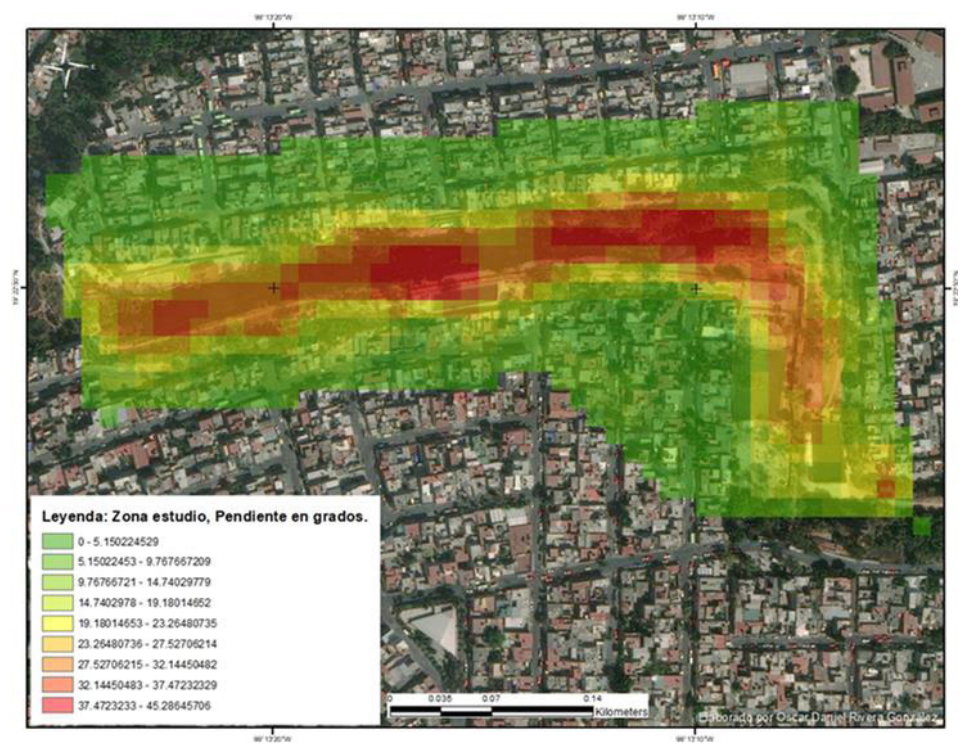

Figura 4. Elaborada con base en datos Raster de la National Aeronautics and Space Administration (NASA). Elaborado con Software ArcGis.

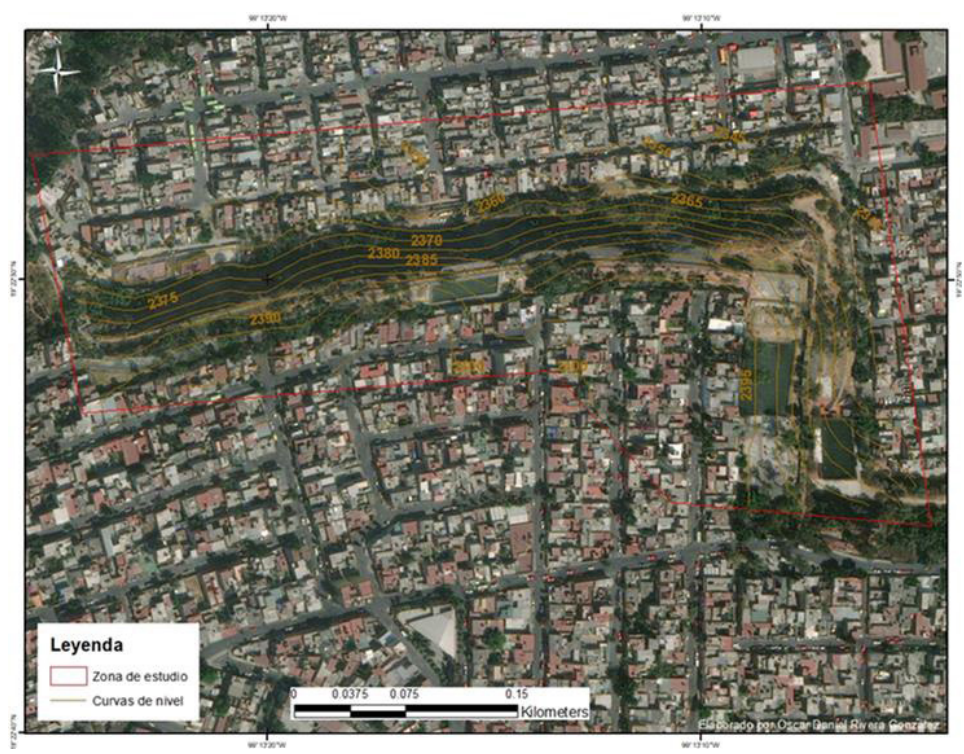

Figura 5. Elaborada con base en datos Raster de la National Aeronautics and Space Administration (NASA). Elaborado con Software ArcGis. 
Existe una Ley general de asentamientos humanos, así como El Programa Nacional de Desarrollo Urbano 2014-2018y la nueva Ley general de asentamientos humanos, ordenamiento territorial y desarrollo urbano, publicadas en el Diario Oficial de la Federación el 28 de noviembre de 2016, mismas que las autoridades competentes, no analizan e implementan.

\section{Metodología}

El trabajo de investigación consistió en la reunión, análisis, recopilación, búsqueda bibliográfica, hemerográfica, periodística y cartográfica de la Alcaldía Álvaro Obregón, trabajo de campo cualitativo, entrevistas con habitantes de la zona de estudio y generación de cartografía con base en encuestas y toma de puntos con GPS. Por otra parte, en cuanto al trabajo de gabinete, se generaron modelos Raster 3D, análisis de fotointerpretación, teledetección y análisis de imágenes satelitales procesadas con softwares ArcGis y QGis para la generación de modelos predictivos que se mostrarán más adelante.

Por otro lado, se buscó información y noticias en páginas electrónicas de periódicos, para identificar y cartografiar zonas de mayor vulnerabilidad que pudieran presentar procesos de remoción en masa, con mayor intensidad en temporada de lluvias y en momentos de sismicidad y la relación que existe con la marginación y nivel socioeconómico de las personas afectadas o posibles afectadas. Con base en lo anterior se tomaron como muestra las cinco colonias antes mencionadas.

\section{Generación de modelos}

Se generaron modelos de predicción para evitar deslizamientos de ladera o remoción en masa dado por procesos gravitacionales.

El primero consiguió generar una gráfica de perfil de la zona de estudio, con base en una imagen Raster de sur a norte de la zona de estudio, donde se observa una mayor vulnerabilidad física para presentar inestabilidad de laderas, en la siguiente figura se muestra un corte vertical sobre el terreno, generado con SIG.

Se generaron tres modelos de elevación visuales, para comprender cuál es la geomorfología del terreno y elevación del mismo, el cual, según su geología, hidrología y edafología, ocasionan procesos de remoción en masa, mismos que afectan a zonas urbanas en la infraestructura de las edificaciones, acarreando la cimentación de las mismas.

En el modelo expresado en la Figura 10 se aprecia el posible proceso gravitacional que se pueda generar en la zona de estudio, posible afectación de viviendas, número de $\mathrm{m}^{3}$ de tierra desplazada y posible expansión del material removido. 

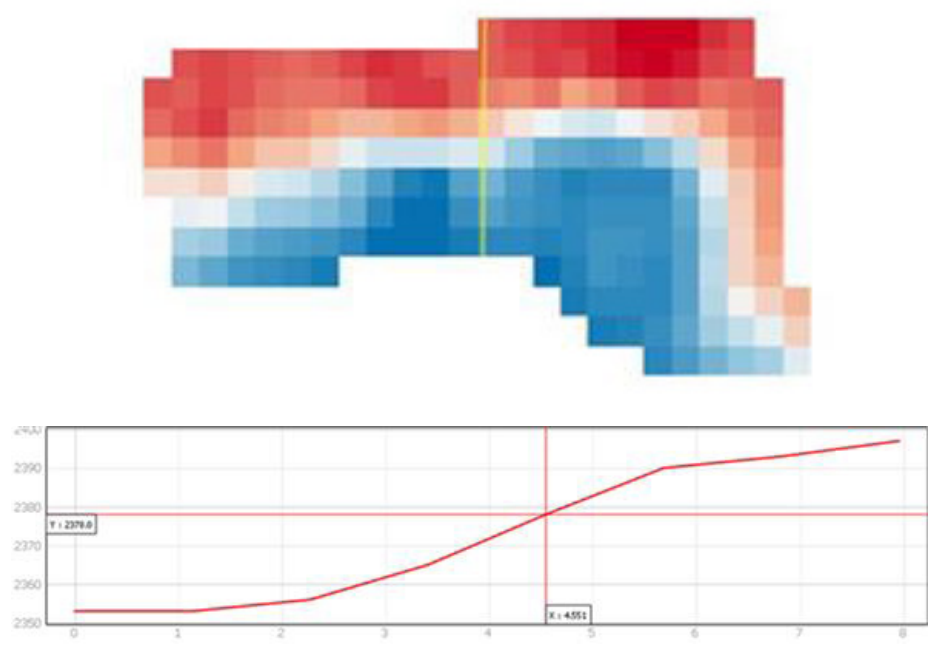

Figura 6. Zona de estudio, modelo digital de elevación (sur a norte) y perfil (izquierda a derecha). Elaborado con Software QGis.
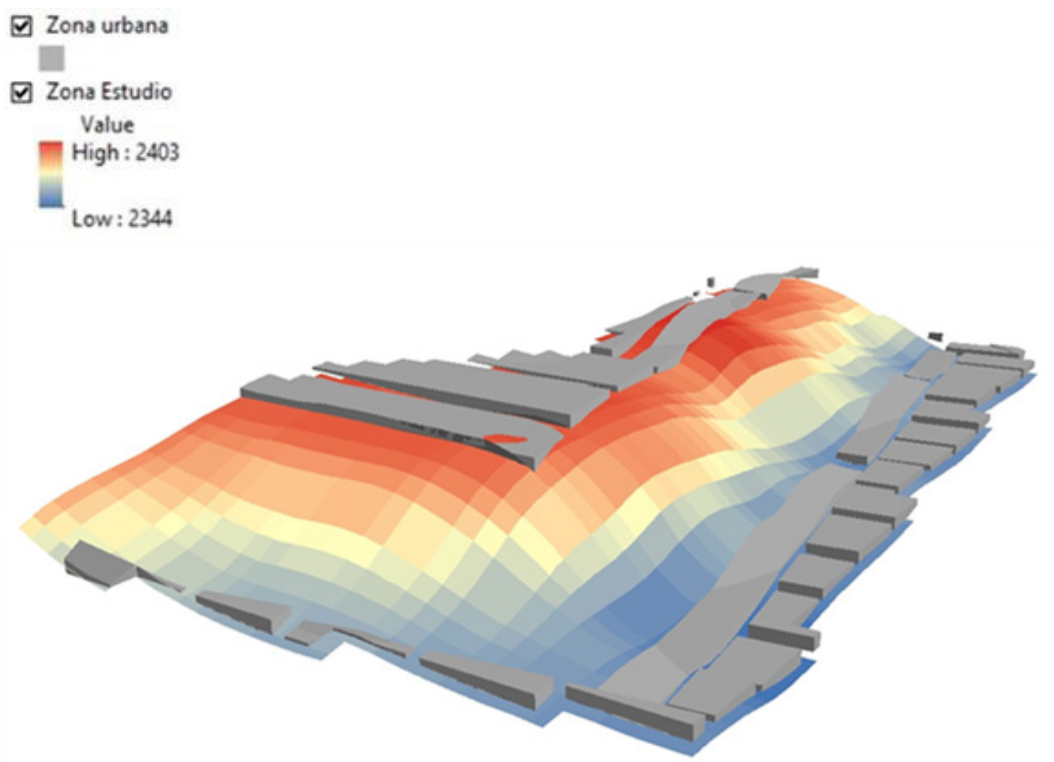

Figura 7. Zona de estudio, modelo digital de elevación 3D. Elaborado con Software ArcGis. 
Zona urbana

Zona Estudio

Value

High : 2403

Low : 2344

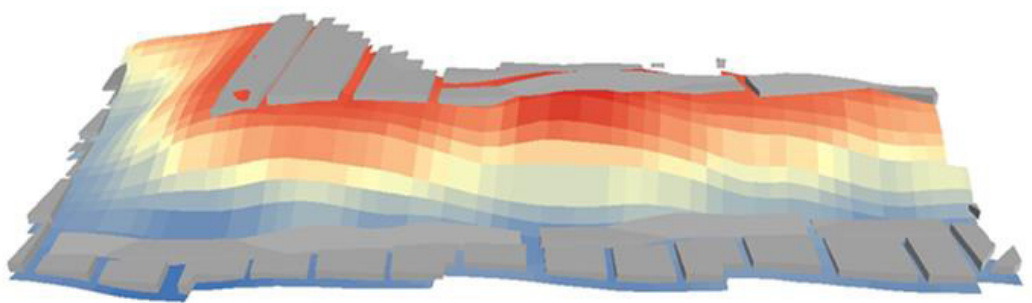

Figura 8. Zona de estudio, modelo digital de elevación 3D.

Elaborado con Software ArcGis.

च Zona urbana

Zona Estudio

Value

High : 2403

Low : 2344

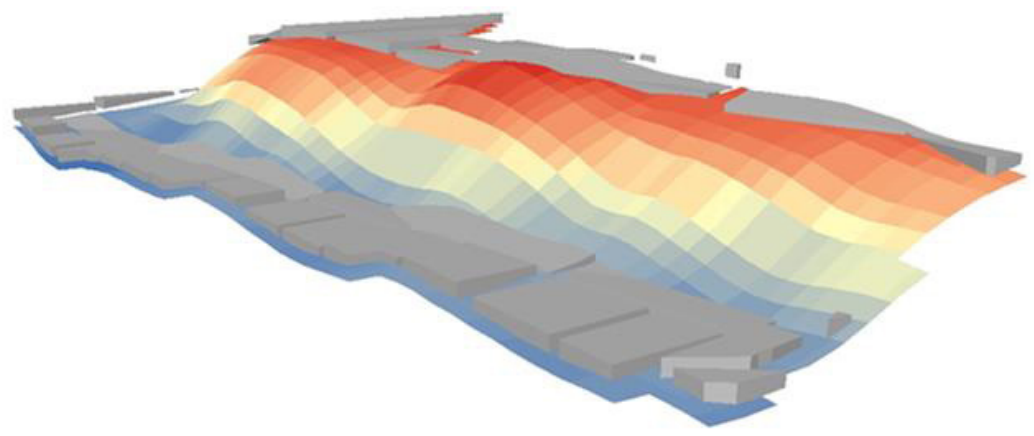

Figura 9. Zona de estudio, modelo digital de elevación 3D.

Elaborado con Software ArcGis. 
Para su mejor comprensión, la estructura de la generación del modelo fue la siguiente:

1. Se generó una triangulación de red irregular (triangulation of the irregular network, TIN), con base en las curvas de nivel obtenidas en el terreno y la zona de estudio (Figuras 10-12).

2. Posteriormente se interpoló el shape o capa de la posible zona de derrumbe, con la triangulación de red irregular (Figura 13).

3. Una vez interpolada la zona de estudio se creó una nueva triangulación de red irregular, que es la que en teoría se removió o existió una posible remoción (observar el cambio de la geomorfología de la zona de estudio (Figuras 11 y 12 contrastarla con las Figuras 14 y 15).

4. Posteriormente se obtuvo la diferencia de superficie, al restar el total de la nueva Triangulación de red irregular (en la cual existe remoción en masa o proceso gravitacional) a la anterior Triangulación de red irregular (la que no tiene remoción en masa o proceso gravitacional), obteniendo el área total de afectación en $\mathrm{m}^{2}$ que se removió, la cual es un área de $74951.73 \mathrm{~m}^{2}$ (Figuras 16 y 17) y el posible lugar donde caerán los $\mathrm{m}^{3}$ de tierra con base en la geomorfología y topografía, la cual es un volumen de $197565.19 \mathrm{~m}^{3}$ (Figuras 18 y 19 ).

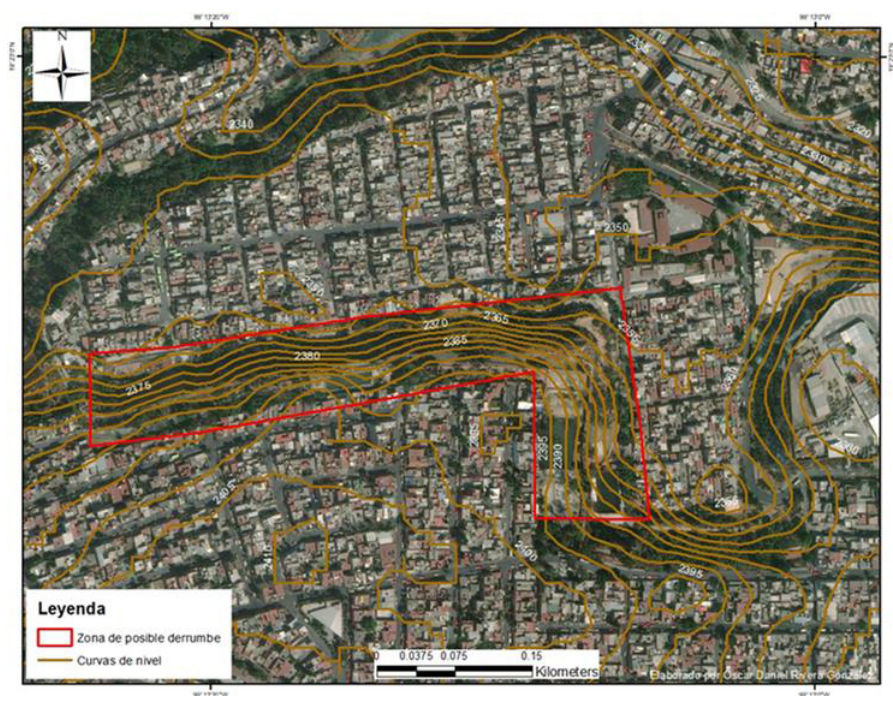

Figura 10. Elaborada con base en datos Raster de la National Aeronautics and Space Administration (NASA). Elaborado con Software ArcGis. 


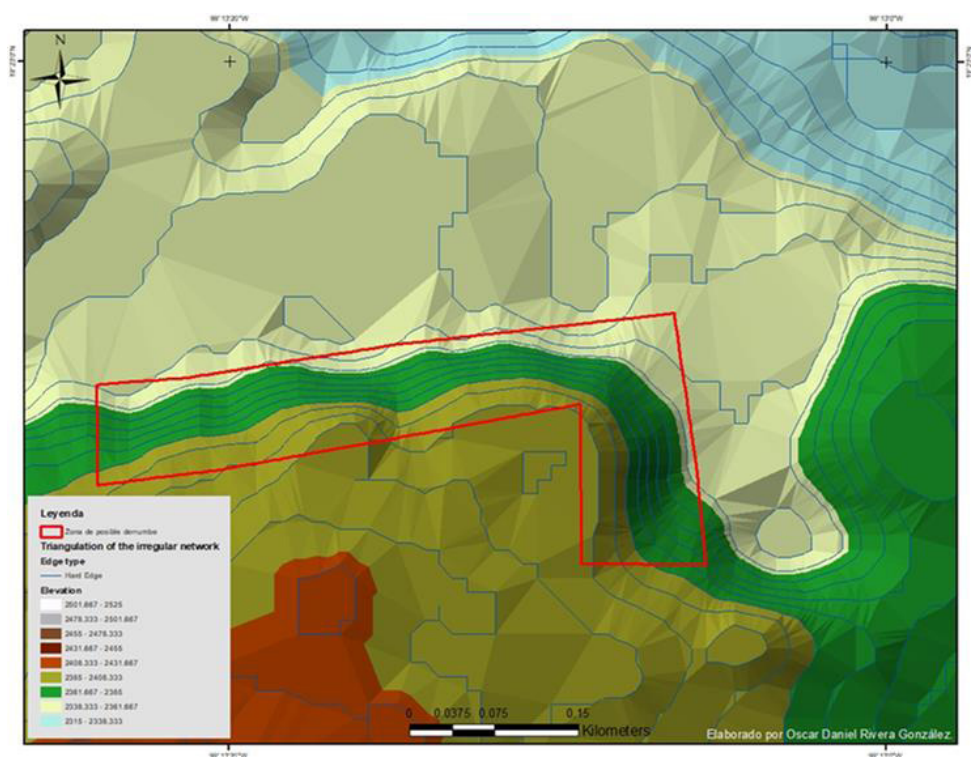

Figura 11. Elaborada con base en datos Raster de la National Aeronautics and Space Administration (NASA). Elaborado con Software ArcGis.

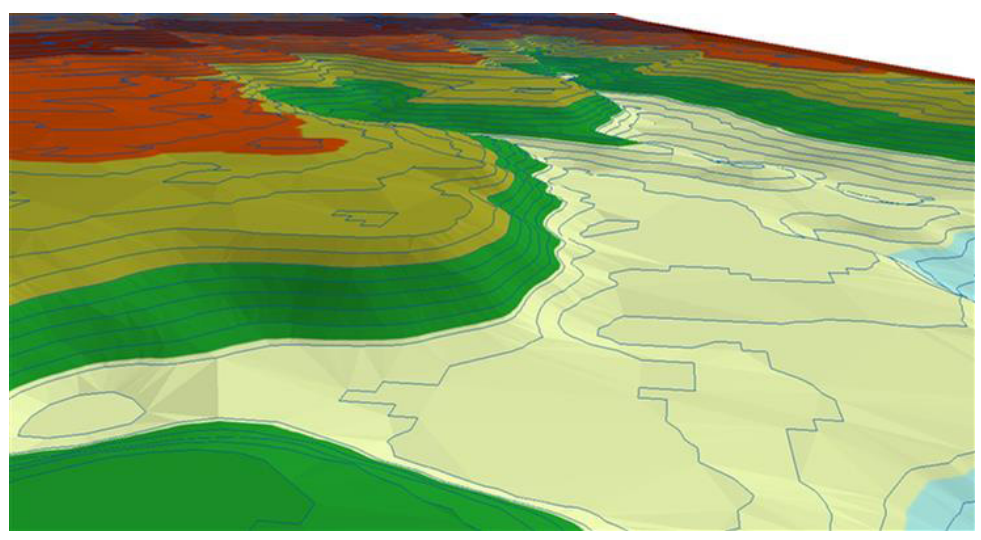

Figura 12. Elaborada con base en datos Raster de la National Aeronautics and Space Administration (NASA), imagen 3D.

Elaborado con Software ArcGis. 


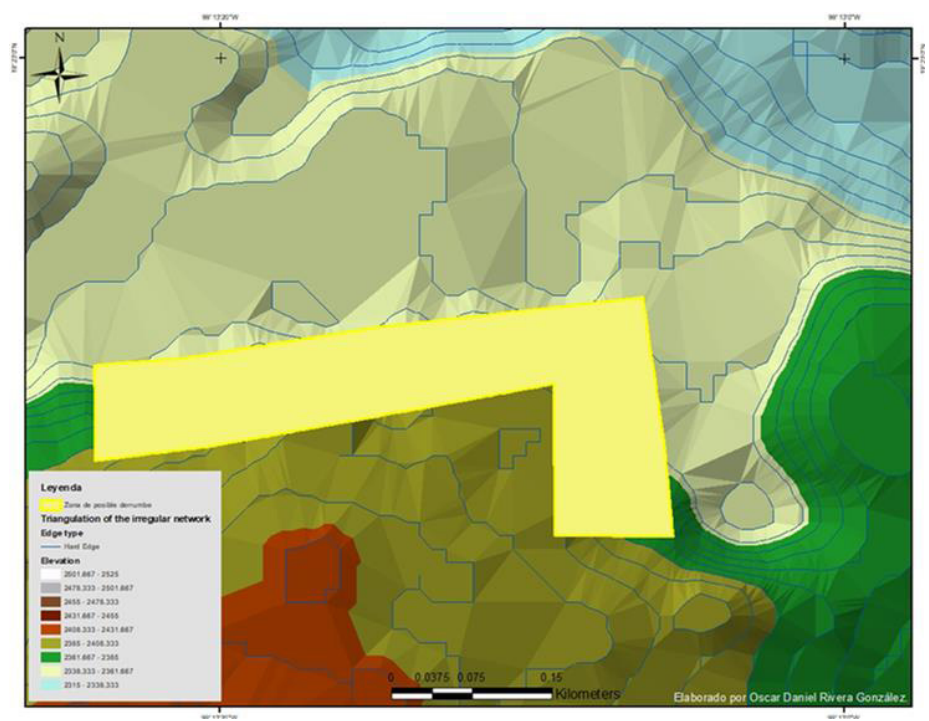

Figura 13. Elaborada con base en datos Raster de la National Aeronautics and Space Administration (NASA). Elaborado con Software ArcGis.

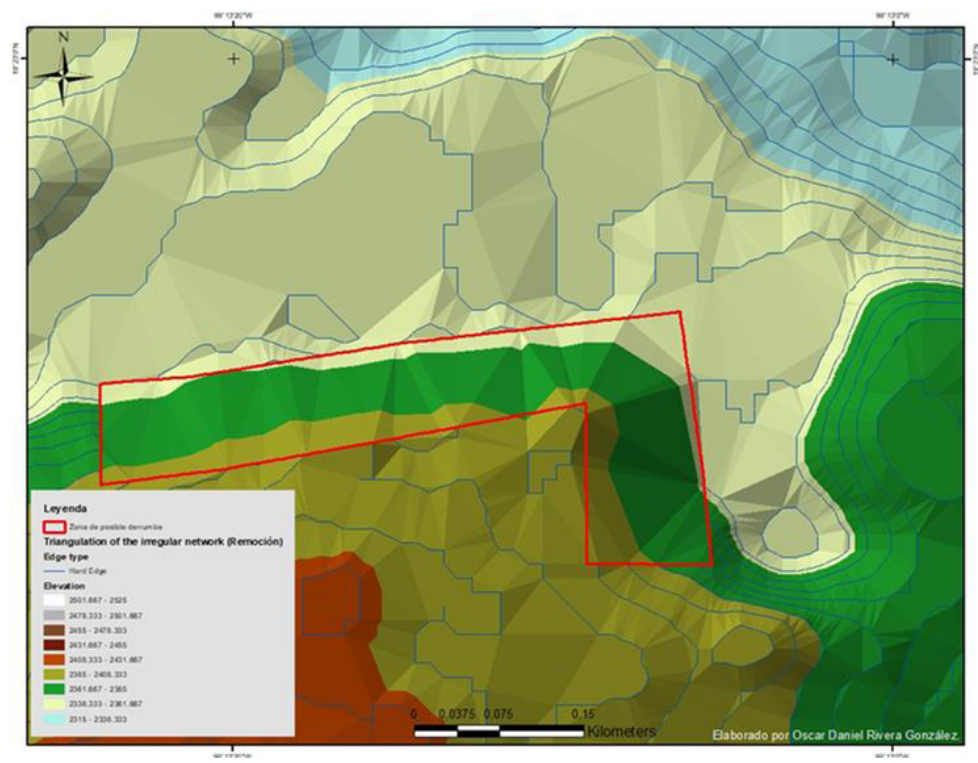

Figura 14. Elaborada con base en datos Raster de la National Aeronautics and Space Administration (NASA). Elaborado con Software ArcGis. 


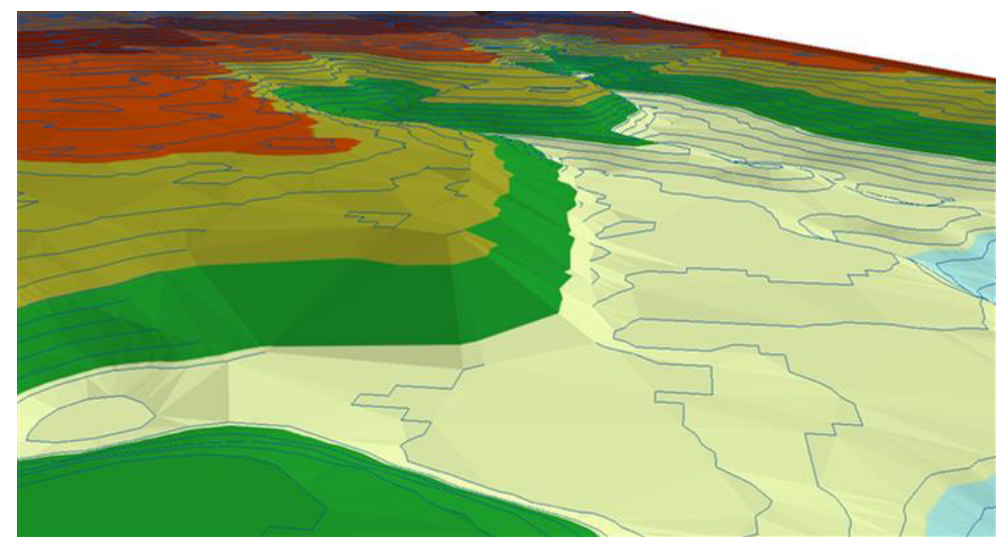

Figura 15. Elaborada con base en datos Raster de la National Aeronautics and Space Administration (NASA), imagen $3 D$.

Elaborado con Software ArcGis.

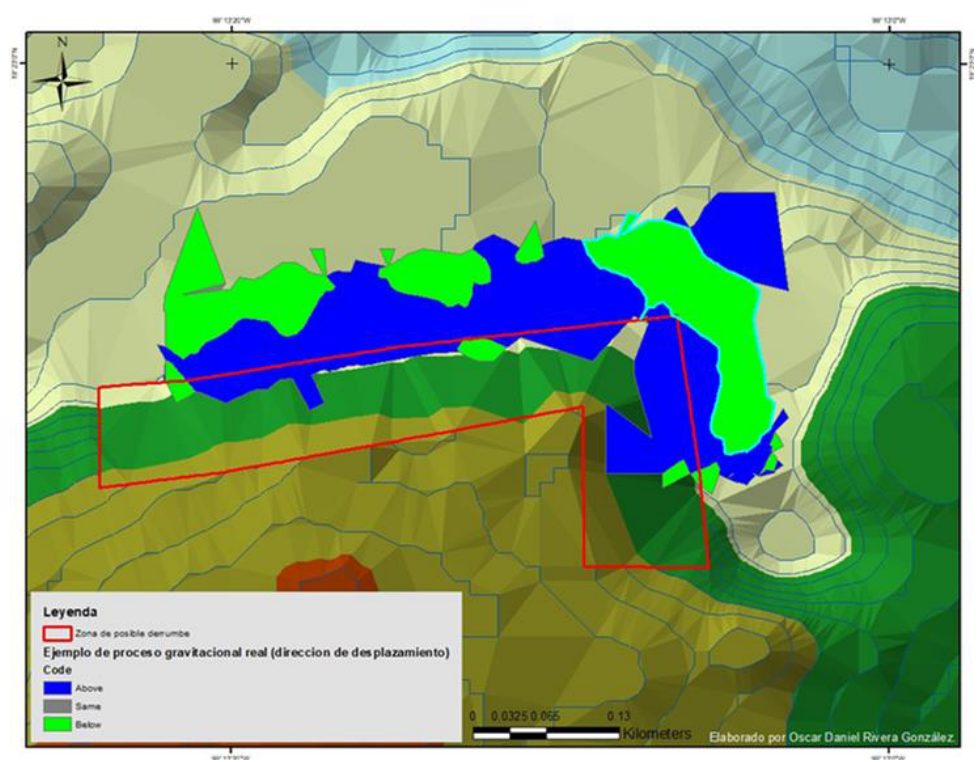

Figura 16. Elaborada con base en datos Raster de la National Aeronautics and Space Administration (NASA). Elaborado con Software ArcGis. 


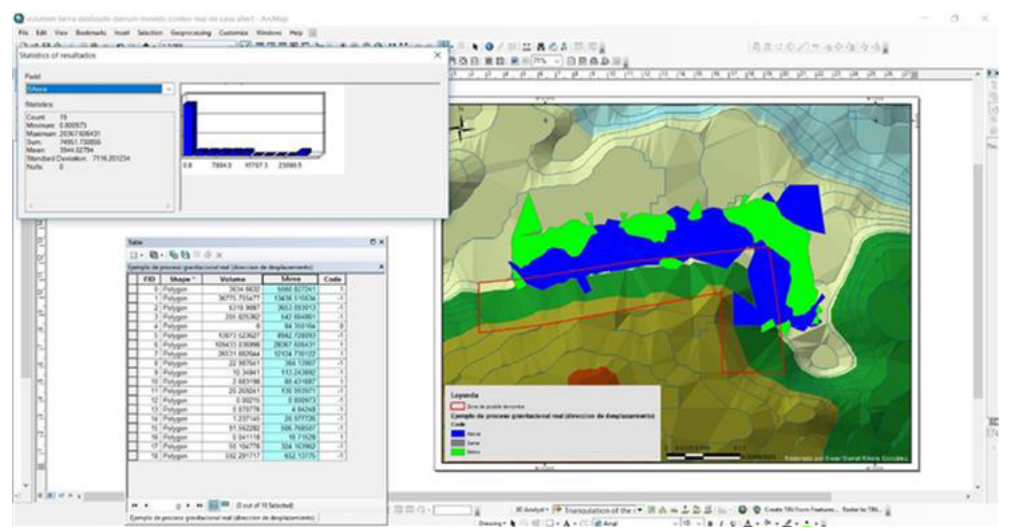

Figura 17. Impresión de pantalla, software ArcGis, elaborada con base en datos Raster de la National Aeronautics and Space Administration (NASA).

Elaborado con Software ArcGis.

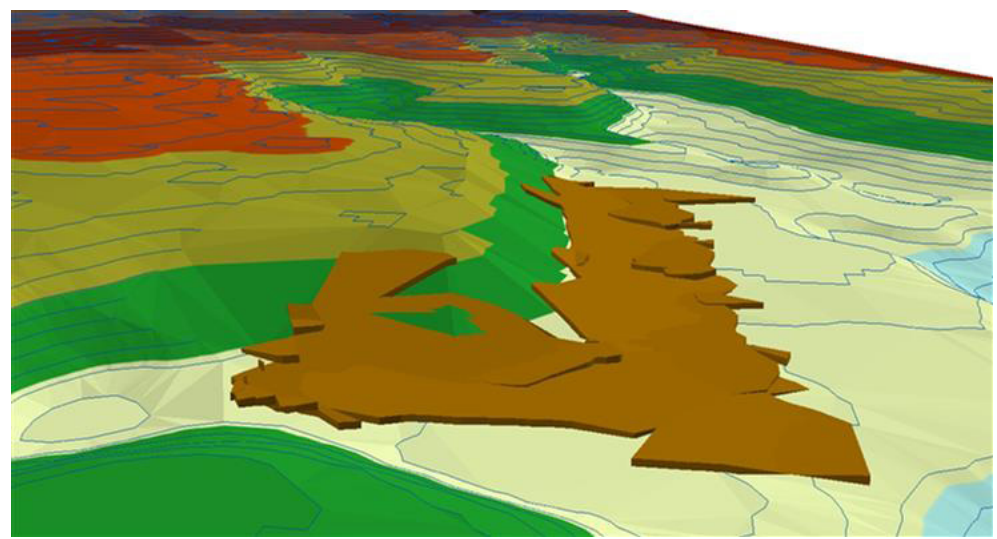

Figura 18. Elaborada con base en datos Raster de la National Aeronautics and Space Administration (NASA), imagen 3D.

Elaborado con Software ArcGis. 


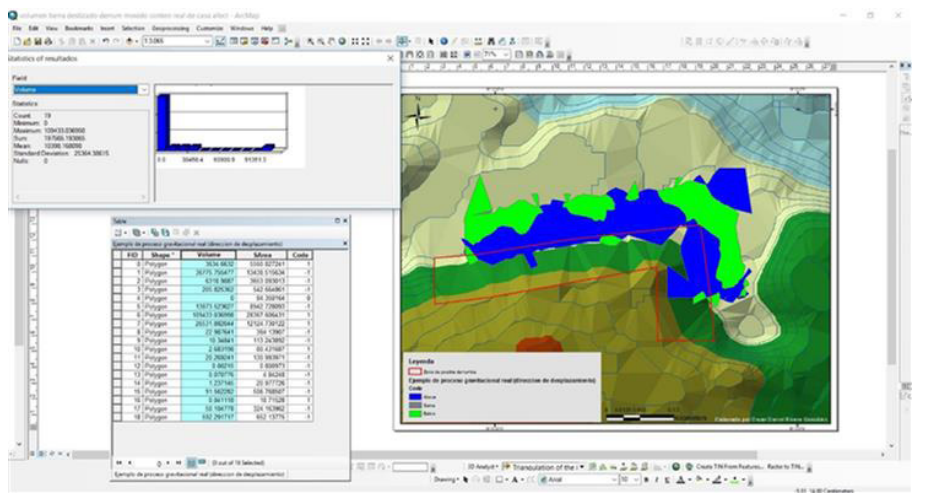

Figura 19. Impresión de pantalla, software ArcGis, elaborada con base en datos Raster de la National Aeronautics and Space Administration (NASA).

Elaborado con Software ArcGis.

\section{Resultados}

Con base en el resutado del modelo predictivo, así como la visita a la zona de estudio, se encontró y definió lo siguiente:

1. Área inicial zona de estudio sin afectaciones: $57049.50 \mathrm{~m}^{2}$ (Figura 11).

2. Área final de afectación (la cual se extendió aún más, por la geomorfología y topografía del lugar muy irregular y accidentada): $74951.73 \mathrm{~m}^{2}$ (Figuras 16 y 17).

3. Volumen total de afectación de deslizamiento: $197565.19 \mathrm{~m}^{3}$ (Figuras 18 y 19).

4. En un sentido urbano y real se verían afectadas: 17 manzanas/cuadras $=136$ casas/construcciones/hogares afectados (Figuras 20-22).

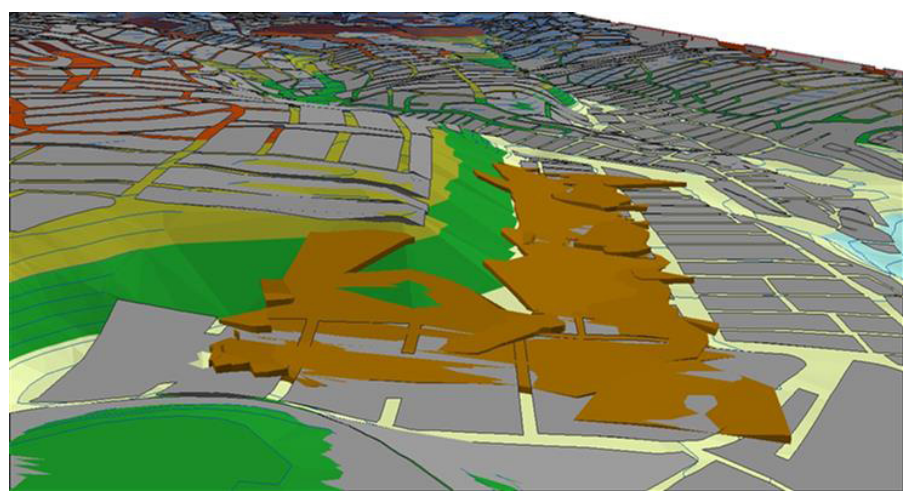

Figura 20. Elaborada con base en datos Raster de la NASA, imagen $3 D$. Elaborado con Software ArcGis. 


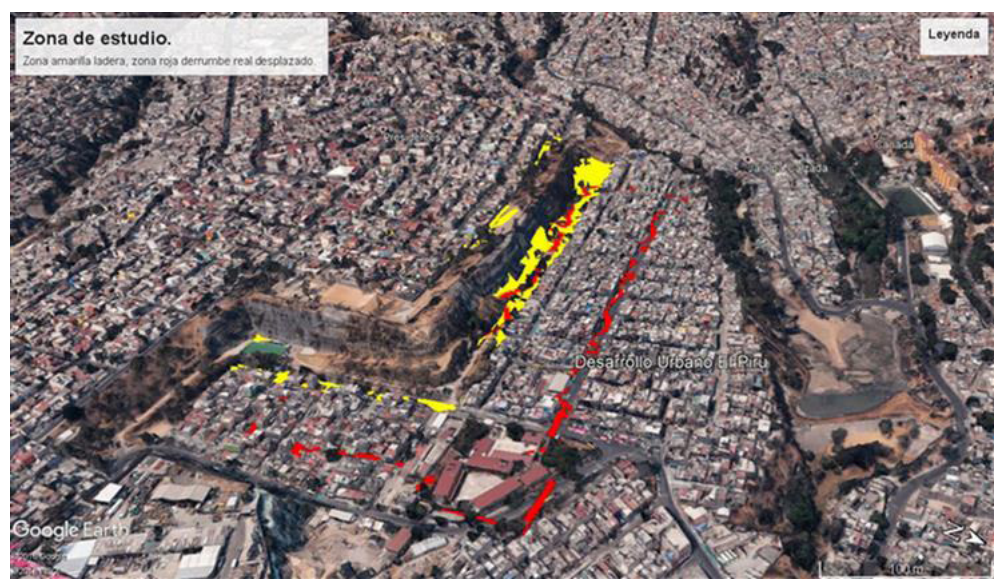

Figura 21. Vista 3D, Google Earth, zona de estudio.

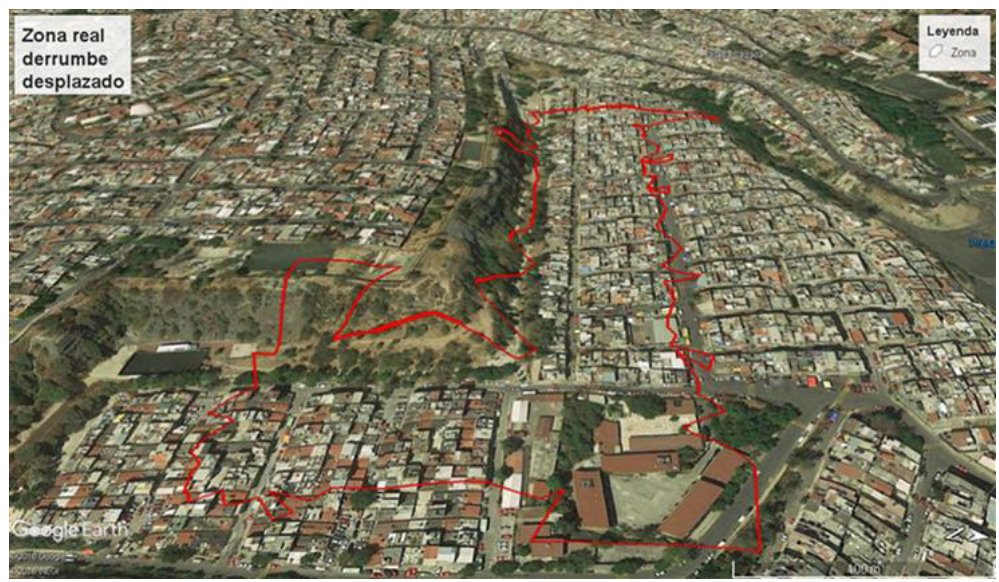

Figura 22. Vista 3D, Google Earth, zona de estudio.

\section{Conclusiones}

La aplicabilidad de modelos de predicción en procesos gravitacionales en zonas de laderas, con base en procedimientos reales y cuantificados como se realizaron a lo largo de este artículo, deben utilizarse en la implementación de políticas públicas para posibles soluciones a problemáticas dadas ante este tipo de episodios geomorfológicos, mismos que indudablemente han acontecido a lo largo de la Alcaldía Álvaro Obregón, existiendo diversas noticias periodísticas que lo avalan, 
por lo que este tipo de modelaciones, pueden aplicarse como posible solución a cualquier zona de dicha alcaldía, Ciudad de México y República Mexicana o inclusive cualquier parte del mundo donde se presenten características, geográficas, geológicas, geomorfológicas, edafológicas, hidrográficas, similares a la zona estudiada en este artículo.

Así mismo, la población afectada y políticas publicas bien gestionadas por parte de los tres niveles de gobierno mexicano, podrían atenuar, aminorar o posiblemente desaparecer diversas problemáticas generadas ante este tipo de episodios geomorfológicos, que cobran vidas humanas año con año.

Por otra parte, sustentar con este tipo de modelaciones, proyecciones de reubicación en zonas urbanas vulnerables a inestabilidad de laderas, concientizando a la población del peligro con el cual viven sus familias a diario, lo que dará como resultado una posible prevención del riesgo y no corrección del mismo, lo que desencadenará disminución o desaparición de problemáticas sociales, económicas, ambientales y físicas, derivadas de este tipo de procesos geomorfológicos.

Por ultimo con este tipo de modelos predictivos, se podrá crear un mapeo de vulnerabilidad baja, media y alta, en zonas de barrancas, mismas que se encuentren urbanizadas, así como replicarlo a nivel Alcaldía, Municipio o Estado, el cual, podría consultar la población directamente en la Alcaldía Álvaro Obregón, o vía electrónica en la página de dicha alcaldía, para que la población posiblemente afectada, sepa cuál es el nivel de peligro con el que vive de manera constante y pueda esta población acercarse a las autoridades competentes y gestionar una posible solución a su petición.

\section{Agradecimientos}

A la Universidad Nacional Autónoma de México, a Kristoff Pineda González y a Oscar Pineda Durán.

\section{Bibliografía}

Alcántara, I., 2016. Investigación forense de desastres: un marco conceptual y guía para la investigación, Editorial Instituto de Geografía, UNAM, México, p. 38.

Instituto Nacional de Estadística y Geografía (INEGI), <http://www.beta.inegi.org.mx/temas/ mapas/relieve/continental/>, consultado el 18 de enero de 2019.

Ley General de Asentamientos Humanos, "Nueva Ley publicada en el Diario Oficial de la Federación el 21 de julio de 1993", Texto vigente, última reforma publicada DOF 30-11-2010, México, 1993, <http://www.senado.gob.mx/comisiones/desarrollo_ social/docs/marco/Ley_GAH.pdf $>$, consultado el 12 de agosto de 2018.

National Aeronautics and Space Administration (NASA), <https: //vertex.daac.asf. alaska. edu/>, consultado el 18 de enero de 2019. 
Nueva Ley general de asentamientos humanos, ordenamiento territorial y desarrollo urbano, publicadas en el Diario Oficial de la Federación el 28 de noviembre de 2016, <http://www.diputados.gob.mx/LeyesBiblio/pdf/LGAHOTDU_281116.pdf>, consultado el 16 de noviembre de 2018.

Programa Nacional de Desarrollo Urbano 2014-2018, <http://dof.gob.mx/nota_detalle. php? codigo $=5342867 \&$ fecha $=30 / 04 / 2014>$, consultado el 14 de noviembre de 2018. 